\title{
Cutting the Path: Encouraging Formal Exploration Through Integration Between Algorithmic and BIM Environments
}

\section{SIGRADI2018 TECHNOPOLITICAS \\ xxii congresso da sociedade iberoamericana de gráfica digital 22th conference of the iberoamerican society of digital graphics 07|08|09|novembro|2018 iau usp | são carlos | sp br}

\author{
Mário Guidoux Gonzaga \\ UFRGS | Brazil | guidoux.gonzaga@gmail.com \\ Leonardo Prazeres Veloso de Souza \\ UFRGS | Brazil | leoprazeres13@gmail.com \\ Angélica Paiva Ponzio \\ UFRGS | Brazil | angelica.ponzio@gmail.com \\ Underléa Miotto Bruscato \\ UFRGS | Brazil | underlea.bruscato@ufrgs.br
}

\begin{abstract}
This paper describes the experience of introducing students to design processes that use algorithm design and BIM tools. An exercise was presented to instigate the students to explore the interface between two known processes: the creation of algorithms in Grasshopper and the manipulation of objects in a BIM environment using Archicad. The exercise aimed at bridging the gap between algorithmic form creation and manipulation and representation and documentation techniques required in the design studios in order to encourage the students to explore new design processes using the appropriate tools at each stage.
\end{abstract}

Keywords: Parametric design; BIM; Grasshopper; Archicad; Workshop.

\section{INTRODUÇÃO}

Segundo Mitchell (2008), mundos projetuais são ambientes em que é possível esboçar possibilidades do mundo real. Desta forma, arquitetos desenvolvem mundos projetuais assim que escolhem as ferramentas que pretendem utilizar em seu processo criativo. É possível criar mundos projetuais em meios analógicos e digitais. Cada um desses ambientes de projeto pode revelar também axiomas não planejados.

Axiomas que, na definição de Mitchell (2008), podem ser considerados como relações que devem acontecer obrigatoriamente em um determinado mundo projetual, tais como limitações físicas dos materiais empregados ou limitações inerentes ao uso da linguagem virtual. Se analisarmos, por exemplo, um mundo projetual criado por um desenho com lápis e papel apenas, evidentemente que a representação gráfica ocorreria de modo bidimensional. Além disso, seria praticamente impossível desenhar uma linha perfeitamente paralela sem o auxílio de instrumentos complementares como esquadros ou réguas "T".

Por outro lado, se estivéssemos trabalhando com maquetes físicas de papel precisaríamos atender à condição de que com o papel é possível obter superfícies retas, dobradas ou curvas, mas jamais seria possível obter uma superfície tensionada, por exemplo. Os axiomas de um mundo projetual estão implícitos nas propriedades físicas dos materiais e nos instrumentos utilizados (MITCHELL, 2008). Assim, para tornar-se capacitado a desenvolver um projeto em um determinado mundo projetual, é necessário que o indivíduo domine suas ferramentas e, por consequência, seus inerentes axiomas.

Antes de o computador tornar-se uma ferramenta usual no cotidiano de arquitetos e designers, o fluxo de trabalho durante um processo projetual delineava-se de modo predominantemente analógico e de maneira contínua, isto é, desenvolvia-se um projeto utilizando apenas mundo, projetuais analógicos.

Com a introdução do computador no processo projetual, o fluxo de trabalho começou a sofrer rupturas, sendo desenvolvido parte em meios analógicos e parte em meios digitais. Com o passar do tempo, as ferramentas computacionais começaram a se estabelecer nas diversas fases de um processo projetual. Contudo, mesmo quando predominam os meios digitais em um processo projetual, o projeto, por vezes necessita migrar entre plataformas, transitando entre softwares de modelagem, de representação gráfica, rendering, entre outros.

Uma vez que a evolução da tecnologia impacta a arquitetura, tanto na sua maneira de ser pensada, como na sua maneira de ser construída, os conhecimentos necessários para os projetistas têm se tornado cada dia mais diversos e estão se modificando para atender às variadas necessidades, adaptações e transformações pela qual está passando a arquitetura (HASKELL, 2016).

Desse modo, o arquiteto que já possuía, por definição, uma característica generalista quanto aos conhecimentos necessários para o seu ofício, agora expande este conceito para as ferramentas que utiliza em seu processo criativo. Todavia, com tantas ferramentas à disposição, 
sejam elas analógicas ou digitais, o processo projetual tornou-se cada vez mais diversificado e fragmentado.

Esta característica é apontada por Branco e Leitão (2017), os autores afirmam que nunca houve tantas opções de softwares para 0 desenvolvimento de projetos de arquitetura, todavia, em seu artigo comentam que a integração entre os softwares disponíveis não possuem um fluxo de trabalho amigável, sendo necessário por vezes, na transição entre um software e outro, recomeçar o modelo desde o início.

Se por um lado o uso de plataformas CAD permite uma maior liberdade no desenvolvimento de formas a partir de suas técnicas de modelagem (BRANCO E LEITÃO, 2017), a adoção de modelos BIM, por outro lado, permite que a documentação bidimensional e a exploração formal tridimensional se desenvolvam simultaneamente, tornando mais dinâmico o processo de projeto arquitetônico e encurtando o caminho entre o modelo virtual e a representação gráfica, possibilitando, dessa maneira, que o fluxo projetual seja contínuo novamente.

Contudo, o uso de modelos BIM em estágios iniciais de projeto, pode inibir o processo criativo. Na plataforma BIM o usuário se vê obrigado a utilizar uma lógica construtiva para o desenvolvimento do seu modelo o que pode resultar em um entrave para um processo criativo que utilize lógicas conceituais diversas (BRANCO E LEITÃO, 2017). Dessa forma vê-se a necessidade de utilizar uma plataforma que consiga integrar estas duas atividades projetuais primordiais: A livre criação de formas e volumes, e a documentação técnica necessária para execução do projeto arquitetônico.

\section{DESIGN ALGORÍTMICO}

Algoritmos são procedimentos para endereçar problemas utilizando uma série finita de passos, que sigam uma sequência lógica e consistente (NATIVIDADE e VENTURA, 2009). Assim, algoritmos não são exclusivamente digitais e podem ser aplicados na resolução de qualquer tarefa cotidiana. Por isso, sua aplicação no âmbito da computação e da arquitetura é de particular interesse neste trabalho.

O design algorítmico, por sua vez, pode ser descrito como a geração de espaços e formas baseados em regras e lógicas descritas de modo algorítmico (TERZIDIS, 2004). Dessa maneira, é necessário que o projetista consiga descrever seu problema projetual em termos algorítmicos, utilizando parâmetros e variáveis para que seja possível obter uma resposta arquitetônica com esse tipo de abordagem. Logo, no que diz respeito ao ensino de técnicas projetuais, é necessário que haja, previamente uma sensibilização dos alunos quanto a este tipo de raciocínio para que seja possível ensiná-los a utilizar tal método de exploração projetual.

A popularização de abordagens algorítmicas em projetos de arquitetura permitiu que arquitetos conseguissem explorar e manipular de maneira cada vez mais eficiente geometrias complexas (FEIST, 2016), assim, o uso de formas complexas em projetos de arquitetura pode ser facilitado pelo uso de sistemas algorítmicos. Outra característica do design algorítmico é a variabilidade. Com o uso de parâmetros definidos por valores numéricos, é possível criar alterações entre as relações geométricas de um determinado objeto e gerar uma grande variedade de versões para uma mesma situação (OXMAN e OXMAN, 2014).

Uma vez que é possível descrever também as condicionantes de projeto de modo algorítmico, esta abordagem permite que o projeto consiga responder a um contexto dinâmico, de maneira responsiva pois, com a alteração dos parâmetros que descrevem suas condicionantes, a volumetria é modificada automaticamente, seguindo as instruções do algoritmo que lhe dá forma.

Todavia, os softwares mais utilizados para modelagem algorítmica em arquitetura não possuem direcionamento para gerar a representação gráfica e documentação técnica necessárias para a execução destes projetos, tais como plantas, cortes, fachadas e detalhes.

\section{BIM}

Em 1972, Ed Catmull desenvolveu a primeira visualização de um elemento tridimensional modelado em um computador. A tecnologia, criada inicialmente para a indústria do entretenimento, foi logo adotada pela arquitetura, levando ao desenvolvimento, nos anos 1980, de softwares de modelagem tridimensional que permitiram aos arquitetos, pela primeira vez, produzir "maquetes eletrônicas".

As maquetes eletrônicas permitiram que pela primeira o arquiteto tivesse a sua disposição todos os pontos de vista possíveis de seu projeto. As perspectivas produzidas no século XIX, mesmo utilizando basicamente os mesmos princípios matemáticos que aquelas extraídas de um arquivo digital, permitiam a visualização apenas de um ponto de vista. Com a criação dos modelos computacionais, foi possível extrair quantas vistas o arquiteto julgasse necessárias para mostrar percursos, relações e proporções de seu projeto. Criou-se, portanto, uma nova categoria de modelos arquitetônicos analógicos:

\begin{abstract}
"Os desenhos e os modelos físicos (maquetes [eletrônicas ou físicas]) são analógicos, uma vez que intuitivamente apresentam características análogas àquelas dos objetos e mostram "aspectos" parecidos quanto à forma visível, às relações geométricas, às dimensões em escala. (MARTÍNEZ, 2000, p. 12)
\end{abstract}

O surgimento dos primeiros computadores pessoais nos anos 1980 e o crescimento exponencial do poder de processamento destas máquinas permitiu o aumento da complexidade dos modelos computacionais. A computação gráfica não precisava mais ser utilizada como apenas um substituto da prancheta nos escritórios. Com a utilização de modelos tridimensionais um escritório de arquitetura pode experimentar formalmente em um ambiente digital e extrair quantas perspectivas ou desenhos técnicos forem necessários para representar o projeto arquitetônico.

O aumento da complexidade dos modelos levou alguns profissionais a questionar se não seria possível inserir informações sobre a edificação no modelo computacional. Foi o que a Graphisoft chamou de Virtual Building, ainda 
nos anos 1980. Pela primeira vez, a representação arquitetônica não era apenas formal: a partir da construção virtual, o modelo analógico citado por Corona Martínez (2000) ganha informações sobre os mais variados aspectos do artefato arquitetônico:

\begin{abstract}
"A informação [contida no modelo] deve englobar as intenções projetuais do arquiteto com respeito ao programa, ao sítio, ao orçamento, aos sistemas do edifício, à performance energética, à estética, às normas de edificação, etc. Para o construtor, o modelo deve incluir métodos de construção, análises quantitativas, informações de custo, cronogramas, simulações da construção, e assim por diante." (LAISERIN, 2003)
\end{abstract}

A construção virtual preconizada pela Graphisoft nos anos 1980 foi agrupada com outros conceitos semelhantes no início dos anos 2000 sob o nome de BIM - Building Information Modeling. A partir da padronização dos protocolos de diferentes softwares tornou-se possível que os escritórios de arquitetura trocassem informação com diferentes agentes envolvidos na concepção e resolução técnica do projeto. Para Laiserin, assim como a implementação dos sistemas CAD automatizou o processo de desenho, o uso do BIM automatizaria a transferência de informações do edifício.

É possível que com a implementação universal de sistemas baseados em modelos contendo informações detalhadas sobre o projeto aconteça uma superação de paradigmas na representação arquitetônica. Se aceitarmos que o modelo análogo ao edifício contém todas informações necessárias para a sua execução física, é possível que superemos a necessidade de extrair desenhos planimétricos:

"Os desenhos serão, pelo menos em um futuro próximo, um produto essencial extraído do $B I M$, mas, em última análise, o valor da informação contida no modelo superará o valor do conjunto de desenhos." (LAISERIN, 2003)

Enquanto tal superação de paradigmas não acontece, os modelos podem ser usados como ferramentas, para que o arquiteto mantenha controle sobre o processo de projeto, atuando como um centralizador da informação.

\section{A-BIM}

De acordo com Sofia T. V. Feist (2016), a combinação entre design algorítmico e a plataforma BIM pode oferecer inúmeros benefícios ao processo projetual em arquitetura. Em sua dissertação de mestrado, a autora classifica essa interação como A-BIM, um acrônimo para "AlgorithmicBased Building Information Modelling". Segundo a autora, alguns profissionais já começaram a explorar essa integração nos últimos anos, mas ainda existe campo para pesquisa e exploração nessa abordagem (FEIST, 2016).

O seu estudo faz um comparativo entre diversas possibilidades de modelagem virtual variando entre modelagens algorítmicas integradas ao CAD, ao BIM e uma modelagem BIM manual. Ao final de sua pesquisa ficou claro que existe uma grande vantagem em se utilizar a estratégia de modelagem A-BIM quando se deseja variabilidade, versatilidade e complexidade ao mesmo tempo em que se mantém o controle dos dados construtivos e se obtém os desenhos técnicos inerentes ao exercício profissional arquitetônico.

\section{CENÁRIO NACIONAL}

Segundo Rufino e Veloso (2005) O ensino de projeto nas escolas de arquitetura passou por modificações devido a introdução de novas tecnologias da informação, entre estas é possível destacar a substituição de instrumentos convencionais de representação gráfica por computadores e softwares que podem até mesmo transcender a mera representação gráfica, a evolução dos equipamentos didáticos tais como projetores e laboratórios de informática, e o uso crescente da internet e do ambiente virtual para comunicação, transmissão e absorção de conteúdo.

Da mesma maneira, o perfil dos alunos que chegam aos cursos de graduação também mudou, tratam-se de indivíduos conectados, habituados com o ambiente virtual, o que permite uma visão espacial diferente daqueles alunos que entravam no curso na década 1990. São indivíduos que já nasceram tendo a internet e os computadores como algo corriqueiro em seu cotidiano, sendo portanto, indivíduos mais familiarizados com a ferramenta computacional.

Todavia as disciplinas de informática aplicadas a projetos de arquitetura desde a sua implementação na década de 1990, possuíam a função de graficação digital dos projetos de arquitetura, e ainda hoje, são vistas por muitos com a função de "passar o projeto a limpo" sendo utilizadas basicamente para a produção de desenhos técnicos (ORCIUOLI, 2010). Assim, é possível afirmar que as potencialidades do uso da informática em projetos de arquitetura estão sendo subaproveitadas nestas disciplinas. Uma das potencialidades que o uso da plataforma digital pode oferecer para o processo projetual é a possibilidade de se trabalhar com modelos paramétricos, o que permite a produção de um modelo dinâmico do partido arquitetônico, passível de se adaptar a diferentes contextos e restrições.

Contudo, de acordo com Romcy (2017) o cenário nacional é ainda incipiente quanto a aplicação de modelos paramétricos no ensino de projeto. Segundo Orciuoli (2010), Na América Latina, e no Brasil, a abordagem de projeto digital é visto com certas ressalvas e preconceitos por parte das faculdades de arquitetura, e com isso, informática não foi incorporada adequadamente na formação do arquiteto, deixando de assumir seu caráter vanguardista e experimental (ORCIUOLI, 2010). Isso torna pesquisas com temas relativos a esta área cada vez mais necessárias e relevantes para modificar a atual situação nas instituições de ensino.

\section{EXERCÍCIO PROPOSTO}

Este trabalho relata a experiência de sensibilização de estudantes de arquitetura em processos de projeto que façam a ponte entre ferramentas de desenho algorítmico e tecnologia BIM. Como parte do projeto de extensão "BIM APLICADO AO PROCESSO DE PROJETO - MÓDULO II", foi proposto, em formato de workshop, um exercício que os instigasse a explorar a interface entre processos até então desconhecidos dos estudantes: criação de algoritmos no software Grasshopper e manipulação do objeto virtual resultante destes algoritmos em ambiente BIM no software Archicad 21. 
Este projeto de extensão foi ministrado no $2^{\circ}$ semestre de 2017 com capacidade para 20 alunos e teve duração de 3 horas durante 5 dias totalizando 15 horas. Por definições internas da universidade o projeto de extensão deveria ser aberto para toda a comunidade acadêmica e também para qualquer indivíduo de fora da universidade que tivesse interesse no conteúdo apresentado, entretanto, todos os alunos matriculados no workshop cursavam arquitetura e urbanismo na UFRGS.

O workshop iniciou-se com 16 alunos matriculados, porém, ao final apenas 8 alunos completaram as aulas e exercícios ministrados. Dentre eles, todos encontravamse, do $4^{\circ}$ semestre em diante do curso e foram capazes de realizar todas as atividades propostas.

O exercício teve por objetivo aproximar a experiência de criação e manipulação de formas com algoritmos às técnicas de representação e documentação exigidas nos ateliês de projeto para, desta maneira, encorajar os estudantes a explorar novos processos de exploração projetual amparados por ferramentas digitais algorítmicas e paramétricas.

O exercício proposto foi o desenvolvimento de uma edificação baseada em um sistema de restrições formais e urbanísticas no ambiente paramétrico para, em seguida, obter os desenhos técnicos na plataforma BIM.

Embora os estudantes já fossem usuários intermediários do Archicad, para muitos foi a primeira experiência com o Rhinoceros 5 e com softwares de modelagem algorítmica.

\section{TERRENOS}

O exercício proposto buscou explorar as consequências formais da utilização de regras paramétricas na geração de formas. Para facilitar o entendimento das relações entre terreno e algoritmo optou-se pela utilização de terrenos de dimensões homogêneas e alguma variação geométrica.

Para isso foram geradas vinte diferentes geometrias de terreno que foram distribuídas a cada participante da oficina de modo que cada indivíduo teria um terreno que, embora único e diferente dos demais, possuía características comuns.
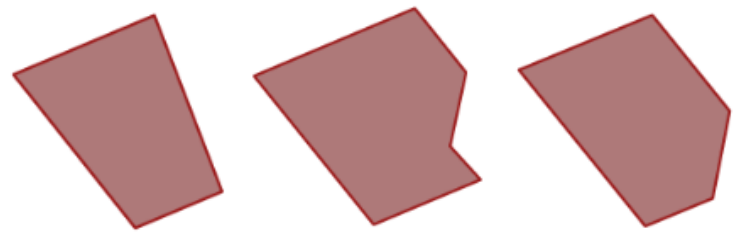

Figura 1: Terrenos de geometrias variadas para cada aluno. Fonte: Autores.

\section{REGRAS VOLUMÉTRICAS}

A exploração formal inicial foi guiada por um conjunto de regras criadas no Grasshopper para simular o regime urbanístico de uma cidade e demonstrar as possibilidades formais do software.

O trabalho iniciou com a definição da testada do terreno, o que definiria o recuo de ajardinamento. Cada estudante pôde testar o recuo de ajardinamento em diferentes lados do seu terreno e visualizar as alternativas.
O próximo passo foi a exploração dos recuos das demais arestas do terreno em decorrência da altura das edificações. Foi arbitrado que estes recuos seriam equivalentes a $20 \%$ da altura total da edificação. Os estudantes foram encorajados a examinar a relação entre a quantidade de pavimentos e a perda de área de planta. Para tanto, os estudantes inseriram um slider - seletor numérico - para a quantidade de pavimentos; este número era então multiplicado por três e o resultado desta operação alimentava o cálculo dos recuos.

\section{EXPLORAÇÃO FORMAL}

Uma vez feita a sensibilização inicial no software e familiarização das técnicas básicas de desenho paramétrico, foi encorajado que os participantes fizessem explorações formais em suas torres com arredondamento de arestas e aumento e diminuição das lajes de cada pavimento.

Nesta etapa, os estudantes puderam pela primeira vez criar um sistema que solucionasse um problema de arquitetura criado por eles mesmos. Não se tratava mais da reprodução de um sistema pronto e sim do encorajamento do pensamento algorítmico.

Uma vez definido o volume externo das torres, os estudantes foram desafiados a lançar o sistema estrutural para seus projetos. Para tanto, foi desenvolvida uma regra que definia uma grelha estrutural regular que cobria todo o terreno e, posteriormente, eram excluídos os pilares que estivessem fora do perímetro dos pavimentos.

O último passo da exploração formal foi o desenho das fachadas. O trabalho iniciou com a criação da superfície externa a partir da interpolação das curvas de cada pavimento com o uso do comando Loft. A fachada foi desenhada pela divisão desta superfície em módulos, de acordo com a divisão de pavimentos, utilizando o plugin Lunchbox - extensão do Grasshopper - que deram origem aos painéis das esquadrias.com variações de aberturas de acordo com sua orientação solar.

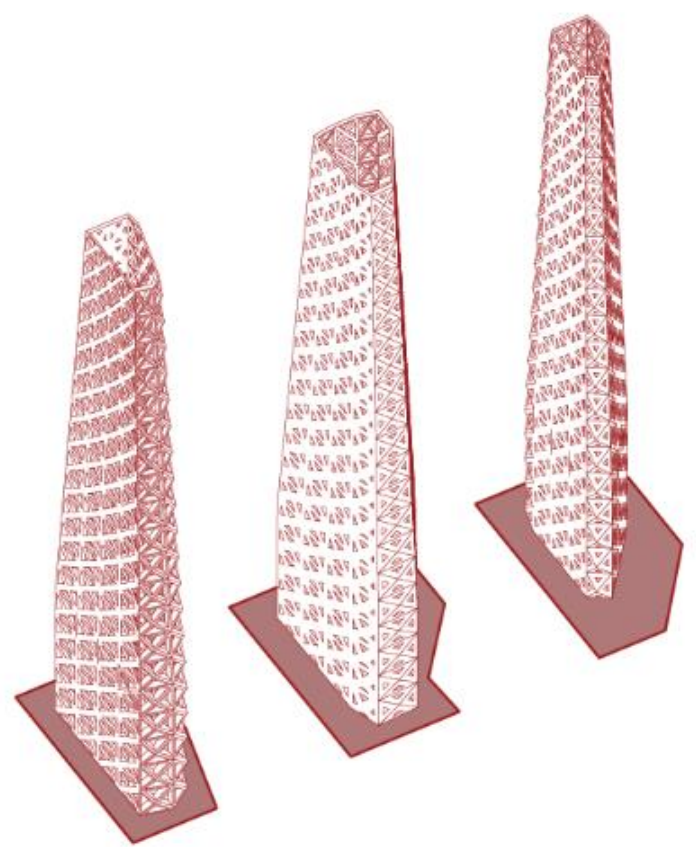

Figura 2: Edifícios diferentes gerados a partir da mesma lógica algorítmica. Fonte: Autores. 


\section{INTERFACE GRASSHOPPER E ARCHICAD}

O objetivo inicial da oficina foi o encorajamento do uso de ferramentas de desenho paramétrico nos ateliês de projeto. Para isso, era necessário que os estudantes pudessem realizar explorações formais complexas ao mesmo tempo que fossem capazes de elaborar desenhos técnicos sem a necessidade de redesenhar seu projeto em outro software. A solução para este problema foi a utilização do plugin Grasshopper Archicad Live Connection que promove a integração entre entre o Grasshopper e o Archicad.

Com isso os alunos tiveram a oportunidade de aprender uma nova maneira de pensar o projeto arquitetônico sem se preocupar com a sua posterior representação gráfica. Dessa maneira, a estratégia projetual pode diminuir a resistência por parte dos alunos em explorar volumetrias complexas em seus projetos de arquitetura.

O plugin permite que a geometria seja criada no Grasshopper de acordo com a linguagem, a descrição de componentes construtivos e configurações nativas do Archicad. Em paralelo, as geometrias geradas são transmitidas simultaneamente para o Archicad mantendo suas propriedades intrínsecas do modelo BIM como material, posição relativa aos pavimentos sem que seja necessário parar a exploração formal, exportar a geometria e importar para o novo software. Isto representa uma continuidade no fluxo projetual dentro do ambiente digital, tornando este processo investigativo mais eficiente

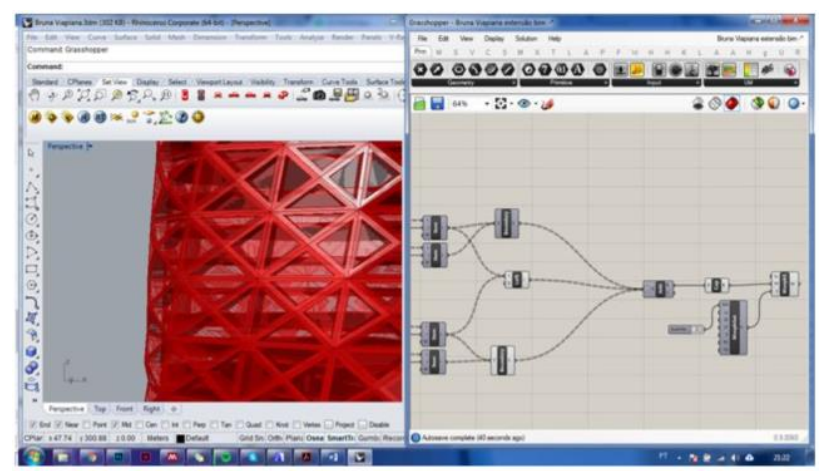

Figura 3a: Modelagem de um edifício hipotético gerada no Grasshopper e Rhinoceros 5. Fonte: Autores.

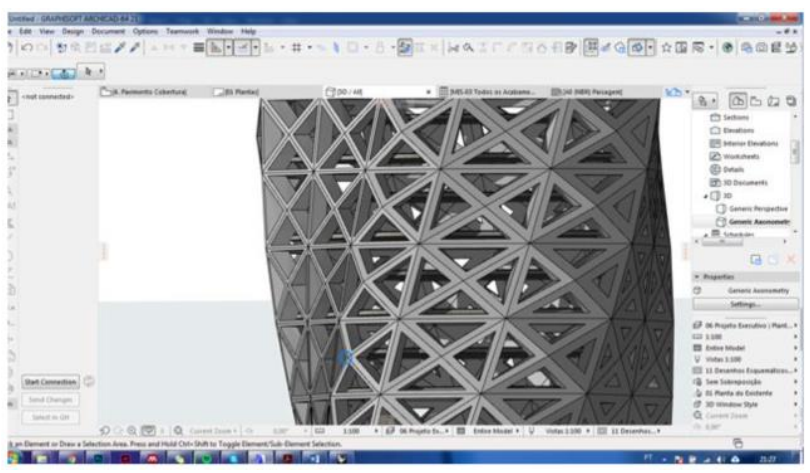

Figura 3b: Modelagem em 3D originada no Grasshopper e transmitida para o Archicad. Fonte: Autores.

Uma vez feita a visualização tridimensional da geometria no Archicad, foram exploradas vistas que o Rhinoceros não permite como plantas de diferentes pavimentos e cortes tanto ortográficos quanto perspectivados. Ao final do exercício os estudantes puderam extrair os resultados de um método de projeto até então desconhecido utilizando uma ferramenta com a qual já estavam familiarizados.

Apesar de representar uma grande inovação, integrando as etapas criativas e técnicas do processo projetual, o plugin apresentou algumas limitações, como pequenos bugs na transmissão de dados e exigindo grande poder de processamento dos hardwares disponíveis no workshop. Todavia, estas questões não representaram um grande problema para a evolução do workshop ou mesmo para o aprendizado dos alunos.

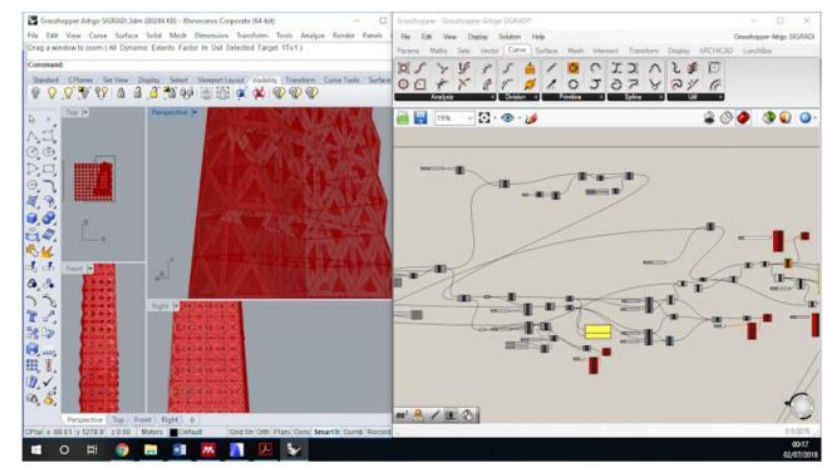

Figura 4a: Modelagem de um edifício hipotético gerada no Grasshopper e Rhinoceros 5. Fonte: Autores.

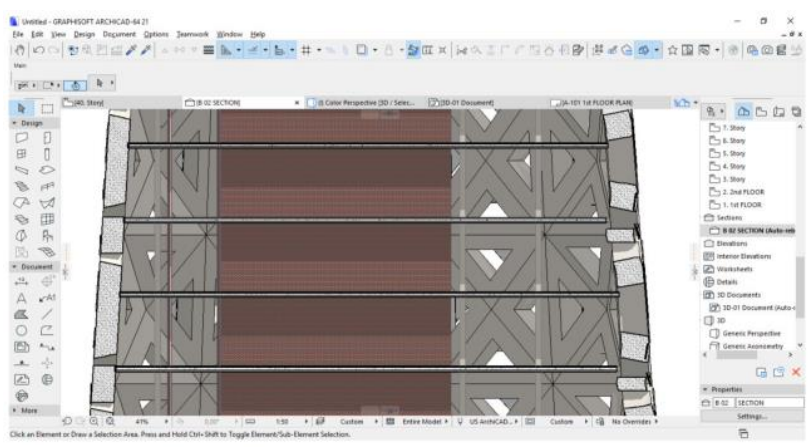

Figura 4b: Corte do edifício obtido no Archicad do modelo gerado no Grasshopper. Fonte: Autores.

\section{DISCUSSÃO}

O sistema de integração entre a plataforma de raciocínio algorítmico digital e a BIM se revelou bastante útil por unir as melhores características de duas plataformas digitais distintas. Contudo, percebeu-se, durante o workshop, que o fluxo de trabalho e a transmissão de dados entre os softwares ainda apresenta problemas de ordem técnica. Por se tratar de um plugin relativamente novo, espera-se que as novas versões do Grasshopper Archicad Live Connection para integrar Archicad 22 e Grasshopper tenha seus "bugs" corrigidos e o desempenho gráfico melhorado.

Ademais constatou-se que a integração proporciona um ambiente favorável para a exploração formal ao eliminar a barreira que outrora existia entre a elaboração de formas complexas e sua representação gráfica e técnica. A capacidade de transmissão simultânea de dados, assim como a opção de atribuir características construtivas às geometrias geradas no Grasshopper, facilita o fluxo de trabalho dos alunos, pois conecta as etapas de projeto que antes aconteciam em momentos distintos do 
processo. Portanto, conclui-se que a integração teve um caráter positivo na experiência de aprendizado dos alunos, possibilitando que eles pudessem desenvolver uma visão global dos processos que envolvem a elaboração de um projeto de arquitetura, além de viabilizar uma diminuição no tempo empregado em realizar tarefas repetitivas, deixando mais espaço para o aprimoramento do raciocínio e da exploração projetual.

O trabalho desenvolvido aponta indícios para um processo projetual que se afasta do paradigma convencional uma vez que apresenta um fluxo de trabalho mais contínuo, desde a etapa de ideação até a etapa de detalhamento técnico, possível de ser realizado em um único mundo projetual, isto é, o digital. Dessa forma, elimina as etapas segregadas e desvinculadas do processo projetual, como croqui analógico, modelagem volumétrica e representação gráfica em plataformas CAD ou BIM. Esta proposta posiciona-se em uma direção divergente ao fluxo de trabalho antigamente empregado no desenvolvimento de projetos de arquitetura, exclusivamente analógico, e se aproxima de uma abordagem projetual caracterizada pelo fluxo de trabalho contínuo e inteiramente digital.

\section{REFERÊNCIAS}

BRANCO, R. C., \& LEITÃO, A. (2017). Integrated Algorithmic Design: A single-script approach for multiple design tasks. eCAADe 35

FEIST, S. T. V. (2016). A-BIM: Algorithmic-based Building Information Modelling. Técnico de Lisboa.
HASKELL, N. (2016, August). Utopía digital: El rol de la competencia material y digital. Materia Arquitectura, 33-39.

LAISERIN, J. (2003). Graphisoft on BIM in: The Laiserin Letter, 2003. Retrieved from http://www.laiserin.com/features/issue19/feature01.php

MARTÍNEZ, A. C (2000). Ensaio Sobre o Projeto. Brasília: Editora UNB.

MITCHELL, W. J. (2008). A Lógica da Arquitetura. Projeto, Computação e Cognição. Campinas: Editora Unicamp.

NATIVIDADE, V., \& VENTURA, A. (2009). Arquitetura Algorítmica. Uma abordagem conceitual. In SIGRADI (pp. 315-317). São Paulo.

ORCIUOLI, Affonso. SIGraDi São Paulo 2009. Quebrando tabus. Drops, São Paulo, ano 10, n. 030.07, Vitruvius, fev. 2010 <http://www.vitruvius.com.br/revistas/read/drops/10.030/2115 $>$.

OXMAN, R., \& OXMAN, R. (2014). Theories of the Digital In Architecture (1a). New York, NY: Routledge.

ROMCY, N. M. S. (2017). Abordagem paramétrica e ensino de projeto - proposição de diretrizes metodológicas, considerando estratégias curriculares e 0 atelier de projeto. UFRN.

RUFINO, I., \& VELOSO, M. (2005). Entre a bicicleta e a nave espacial-os novos paradigmas da Informática e o ensino do Projeto Arquitetônico.

TERZIDIS, K. (2004) Algorithmic Design: A Paradigm Shift in Architecture? p. 201-207. 